\title{
8. EINRICHTUNG DER TEXTAUSGABE
}

Ziel der folgenden Editionen ist eine möglichst buchstabengetreue Wiedergabe, was angesichts des guten Formniveaus der Handschriften bzw. einer gedruckten Überlieferung leicht erreichbar scheint. Als mögliche Hinweise auf Schreib- und Lehrpraxis wurden beim Holubar̆glossar A sogar alle Merkstriche ab 52r bzw. das Fehlen von Auszeichnungspunkten vermerkt, s. dazu S. 17 f. und S. 33. Trotzdem stellen sich bei der Textedition die üblichen Unschärfen ein. So ist die Unterscheidung von c und t, vor allem in Verbindung mit z, mitunter schwierig ${ }^{273}$, ich habe sie nach bestem Wissen gelöst und von einer Vereinheitlichung Abstand genommen ${ }^{274}$, weil erkennbare Fehler wie Bursa/peicel statt peitel (Cod. Pal. lat. 1787 = Holubařglossar A, 55 ${ }^{r}, Z$. 4) Rückschlüsse auf den Schreiber erleichtern können. In gleicher Weise wurde das Problem der Groß- und Klein- sowie der Distinktschreibung behandelt: Es kann ja von Bedeutung sein, wenn - wie im Trialogus (13' Z. 20) - offensichtlich nicht erkannt wird, daß die tschechische Kolumne für Porrus/lauch zwei Synonyme por und luk umfaßt, die für ein Wort porluk genommen werden. Die Fehlform Erweiz im CVP 2945 wird nur nachvollziehbar, wenn wir berücksichtigen, daß das Holubar̆glossar für Orphanus Enweiz, also Nomen und Artikel in einem schreibt (33', Z. 14), was der schlichte Kopist eben umdeutete.

Nur in wenigen Fällen hat unsere Edition Buchstaben - wegen graphematischer Irrelevanz - vereinheitlicht: positionsbedingt langes s wird als s geschrieben, die beiden r-Formen sind als r normalisiert. Diakritische Zeichen sind, soweit als überschriebenes e noch nachvollziehbar, generell durch Doppelpungierung gekennzeichnet, weitere Erklärungen folgen punktuell zu den einzelnen Handschriften. Darüberhinaus hat der Kopist des Trialogus y durchgehend mit Doppelpunkt überschrieben und die deutschen Diphthonge au, eu, gelegentlich

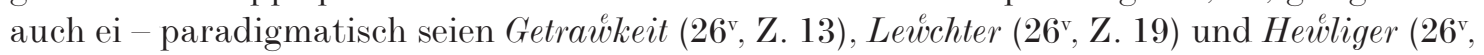
Z. 20) genannt - mit diakritischem e über dem w realisiert. Auch diese reinen Formalismen sind indistinkt. Daher konnte auf eine diakritische Wiedergabe verzichtet werden. Überschreibungen auf Buchstaben, die als nachträgliche Einfügungen in die einzelnen Texte erkennbar sind, wurden nur als Fußnote gekennzeichnet.

Sonst aber gilt den für den Linguisten so wichtigen Diakritika besonderes Augenmerk, und diese Ausgabe soll nicht hinter dem hohen Niveau von Zahradniks Edition zurückbleiben $^{275}$. Generell gilt, daß Überschreibungen von allen Kopisten sparsam und keineswegs konsequent in den deutschen Kolumnen verwendet werden, etwas weniger häufig auch in den tschechischen Texten.

Beginnen wir mit dem Holubar̆glossar A, Cod. Pal. lat. 1787: Hier dienen vereinzelt Häkchen, Doppelpungierungen und akzentähnliche Diakritika zwischen den Buchstaben zur

\footnotetext{
273 Unfreiwillige Belege bieten die verschiedensten Mittellatein-Lexika.Vgl. u.a. auch E. SkáLA, Das Prager Deutsch des 16. Jahrhunderts in: Festschrift für Hans EgGens zum 65. Geburtstag (= Beiträge zur Geschichte der deutschen Sprache und Literatur 94). Tübingen 1972, 288; M. RoQUEs, Recueil général des lexiques français du moyen âge (XIIe - XVe siécle) 1. (Bibliothèque de l'Ecole des Hautes Etudes. Sciences historiques et philol. 264) Paris 1936, XXII.

274 S. dagegen Moser, Kanzlei Maximilians (zit. A. 269) 125.

275 Zahradnik, Slovník (zit. A.7).
} 
Anzeige der „Weichheit“, vgl. A Zěn (29v', Z. 14, tschechisch žeň), Jabllön (59', Z. 16, tsche-

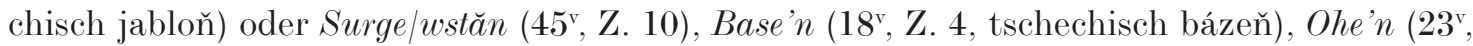
Z. 10, oheň) u.ä. Außerdem gibt es vereinzelt Punktierung ober, ganz vereinzelt auch unter

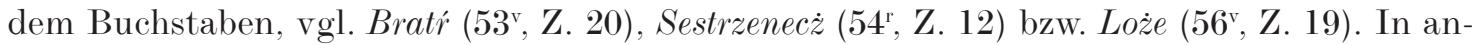
deren Fällen, wo unvermutet gehäuft Überschreibungen auftreten hat man den Eindruck, der Schreiber habe sich plötzlich einer orthographische Neuerung besonnen, auf die er dann wieder vergaß. So gibt es zweimal hintereinander Punktationen für den modernen Hatschek

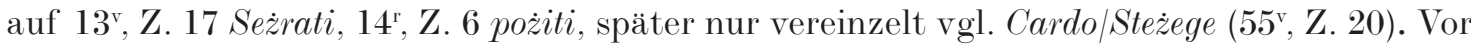
allem auf den letzten Seiten wird Vokallänge über y nicht selten mit einem Strich (čarka) angedeutet: vgl. Chudý, Bohatý, Hrdý (65' Z. 2. 3. 15) bzw. darebný (48v, Z. 13) u.a.

Die Kopie des Holubařglossars im CVP 2945 (B) bringt - v. a. im tschechischen Teil häufig und variantenreich überschriebenes y etwa als y mit zwei Punkten, aber auch Schrägund Querstrich, vgl. Abb.33. Diese werden sinngemäß mit $y$ bzw. $\ddot{y}$ markiert. Überraschenderweise kennt B - mit Ausnahme von Dobrzie (8, Z. 2) - keine Kennzeichnung für Erweichungen. Demgegenüber existieren im vergleichbaren Mosbach-Vokabular des Cod. Pal. lat. 1789 gelegentlich schon ř, $\check{z}$, č̌-Umsetzungen ${ }^{276}$. Der Trialogus hat in der slawischen Kolumne überhaupt keine Diakritika. Vom bemerkenswerten Sonderfall des gedruckten Dictionarius trium linguarum von 1513 war bereits im vorvorigen Kapitel die Rede.

In den deutschen Kolumnen unserer Manuskripte bezeichnen Überschreibungen - wie zu erwarten - v. a. Umlaut und sind entweder stilisierte e-Diakritika, im Trialogus meist kreisförmige Punktierungen, die mit Umlautzeichen wiedergegeben werden, oder einfache Akzente, vgl. etwa in der Holubařüberlieferung A Gärbe B Gärb (18 $\left.{ }^{\mathrm{v}}, \mathrm{Z} .19\right)$, A Öm B Óme (4 ${ }^{\mathrm{r}}$,

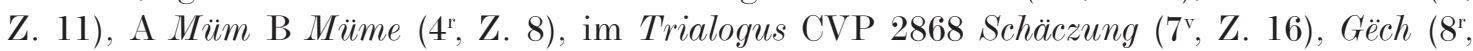
Z. 14), Schöpfe (8v Z. 3), Bürgschafft (7v, Z. 11), Fürdrung (7v, Z. 24) usw., wobei es vereinzelte Mißverständnisse zu geben scheint, vgl. Cingula/satlgürt (12' Z. 6, im Druck satelgurt), Latro/ Vörbörg (42 $2^{\mathrm{v}}$, Z. 11), im CVP 2945 (B) Ein tör $\left(24^{\mathrm{v}}\right.$, Z. 16). Im CVP 2945 (B) scheint $\ddot{a}$ darüberhinaus Schwachtonigkeit anzuzeigen, vgl. Adäler (4 ${ }^{\mathrm{v}}, \mathrm{Z}$. 8) oder altär $\left(6^{\mathrm{v}}, \mathrm{Z}\right.$. 16), dazu im Trialogus Seminator/Seër (11 $\left.{ }^{\mathrm{r}}, \mathrm{Z} .14\right)$, Keslër, Schüslër (10 ${ }^{\mathrm{r}}, \mathrm{Z} .5$ bzw. 7), bzw. - nur im Trialogus - auch ü, vgl. Gefanngknüs (8', Z. 5), Gleichnüss (26r, Z. 27). Cod. Pal. lat. 1787 (A) verwendet $\ddot{u}$ darüberhinaus zur Kennzeichnung des mhd. Langvokals û, vgl. hüte B haut (35', Z. 11), u. a., vgl. die Beispiele auf S. 35. Vielleicht hängt damit die nur zögerliche Umlautfixierung zusammen, vgl. A Rucke B Rücke (15, Z . 15) u. a. Vereinzelt wird ü auch zur Kennzeichnung von mhd. uo genützt, vgl. A Furfüs $\left(4^{\mathrm{r}}, \mathrm{Z} .14\right)$, B zütutler $\left(6^{\mathrm{r}}, \mathrm{Z} .12\right)$, frü oder Phlügstercz u.a. im Trialogus (1 $\left.{ }^{\mathrm{r}}, \mathrm{Z} .25,12^{\mathrm{r}}, \mathrm{Z} .11\right)$. Gelegentlich hat CVP 2945 auch Markierung von alter und neuer Vokallänge: Penitus/Gárr (!) (35, Z. 18), Schád (35v , Z. 11), Sée (44v , Z. 8), vgl. im Holubařglossar A Eppfelbóm (59' ${ }^{r}$, Z. 16). Man vgl. dazu im Trialogus sëligmacher (26 $\left.{ }^{\mathrm{v}}, \mathrm{Z} .17\right)$, eventuell auch genëm $\left(27^{\mathrm{r}}, \mathrm{Z}\right.$. 1) u.a. Die in allen Texten nur spärlichen Interpunktionen wurden berücksichtigt, Abbreviaturen sind in den folgenden Wiedergaben in runder Klammer aufgelöst.

In der Edition des Holubar̆glossars wurden dem Leittext des Cod. Pal. lat. 1787 (=A) in jeder Kolumne des Abecedariums die Lesarten der Kopie CVP 2945 (=B) kursiv beigefügt. Zwecks besserer Übersicht über den Textablauf ist, zusätzlich zu den Blattnummern von A auch die Folienzählung von B festgehalten.

Bei der Ausgabe des Trialogus, CVP 2868 wurde - aufgrund größerer Abweichungen im Kontext - neben die Wortliste der Handschrift parallel der gesamte Wortlaut des Dictiona-

${ }^{276}$ Vgl. KR̆eś́lková, Vocabolario Quadrilingue (zit. A. 221) XXXVII-XXXVIII. 
rius trium linguarum schräg gesetzt und zwar in der Reihenfolge der Handschrift, die auch allein Blatt- und Zeilenzählung bestimmt ${ }^{277}$. Um diese buchstäbliche Breite der Tradierung unterbringen zu können, wurde ausnahmsweise querformatig gedruckt. Die einer Überlieferung zunächst scheinbar fehlenden Zeilen tauchen bei der anderen meist an anderer und nicht weit entfernter Stelle wieder auf. Die diesbezüglichen Verweise sind in der jeweils leeren Zeile eingefügt. Interpolationen des Druckes sind mit einem nach und der Nummer der letzten vorangegangenen Trialoguszeile gekennzeichnet. Im Zusammenhang ist auch die mittelbare Textquelle berücksichtigt: Jeweils an den Zeilenenden steht die Nummer der entsprechenden Zeile des Klaretvokabulars nach der Ausgabe von Flajšhans ${ }^{278}$, bei Wortvarianten, die noch immer den Zusammenhang erkennen lassen, in runder Klammer. Die lateinischen oder tschechischen Abweichungen des Klaret sind in den Fußnoten vermerkt. Auch Boh. für Bohemarius bzw. Vokabulař grammatický beziehen sich hier immer auf den zweiten Band der Edition von Vaclav Flajšhans.

Anmerkungen sind so sparsam und kurz wie möglich gehalten. Dabei werden Kurzzitate, also etwa Diefenbach, Grimm, Jungmann, Schmeller usw., bzw. die bekannten Siglen StčSl ${ }^{279}$, BöW ${ }^{280}$ verwendet. Genaue bibliographische Angaben sind im Verzeichnis der abgekürzt zitierten Literatur nachzulesen.

${ }^{277}$ Zum Vergleich der Textabfolgen dient die Konkordanz auf S. 60 ff.

278 FlajšHans (ZIT. A. 75).

279 Staročeský slovnik. Über Pläne und derzeitigen Stand des Alttschechischen Wörterbuches referiert M. Hoмolкová, Möglichkeiten der Erkennung und Beschreibung lexikalisch-semantischer Strukturen (am Beispiel des Alttschechischen). Wiener Slawistisches Jahrbuch 46 (2000) 183.

${ }^{280}$ Wörterbuch der bairischen Mundarten in Österreich. Hrsg. vom Institut für österreichische Dialektund Namenlexika (Bayerisch-österreichisches Wörterbuch I. Österreich) Wien 1963 ff. 